\title{
Optimization Analysis of Correction Tape Box Compounding Cavity Runner Balance Based on Moldflow
}

\author{
Hongbing Wang ${ }^{1,2}$, Chunhua Sun ${ }^{1,2}$ \\ ${ }^{1}$ Department of Mechanical \& Electrical Engineering, Suzhou Vocational University, Suzhou, China \\ ${ }^{2} 3 \mathrm{C}$-Product Intelligent Manufacturing Engineering Technology Research and Development Center of Jiangsu Province, \\ Suzhou, China \\ Email: whb@jssvc.edu.cn
}

How to cite this paper: Wang, H.B. and Sun, C.H. (2020) Optimization Analysis of Correction Tape Box Compounding Cavity Runner Balance Based on Moldflow. World Journal of Engineering and Technology, 8, 42-49.

https://doi.org/10.4236/wjet.2020.81005

Received: January 7, 2019

Accepted: January 7, 2020

Published: January 10, 2020

Copyright $\odot 2020$ by author(s) and Scientific Research Publishing Inc. This work is licensed under the Creative Commons Attribution International License (CC BY 4.0).

http://creativecommons.org/licenses/by/4.0/

\begin{abstract}
Aiming at the problem of runner unbalance in the compounding cavity of the upper and lower cover of the correction tape box, the plastic injection analysis software Moldflow was used to optimize runner balance. First, the 3D modeling software Proe was used to establish the geometric model of the upper and lower cover of the correction tape box, and introduced into the plastic injection analysis software Moldflow. Secondly, the upper and the lower cover of the correction tape box were meshed and the initial gating system was designed in Moldflow. Filling analysis of the initial scheme of the correction tape box combined cavity showed that the runner of the melt was not balanced in the mold cavity. Finally, the runner balance optimization analysis of the cavities was carried out. Through optimization, the time unbalance rate of the melt in the mold cavity decreased from $28.6 \%$ to $0.7 \%$, the pressure unbalance rate decreased from $42.0 \%$ to $4.2 \%$, and the pressure distribution in the cavity was more uniform in the whole injection process.
\end{abstract}

\section{Keywords}

Compounding Cavity, Runner Balance, Optimization

\section{Introduction}

Compounding cavity mold refers to the production of two or more different plastic products in a single die, which can reduce the cost of the die and improve efficiency. It is widely used in the production of the die. In the production of compounding cavity injection, it is necessary to consider the balance of melt in the runner system. Balanced runner system can not only improve product qual- 
ity, but also ensure the consistency of product quality in different cavity [1] [2].

The traditional design method is to achieve runner balance by constantly manually modifying the size of the gating system. This method not only takes time, but also improves the production cost. With the development of CAE technology, the experiment of the mold can be replaced by Moldflow simulation software and the efficiency can be improved [3] [4].

In this paper, the runner balance design of the correction tape box compounding cavity is analyzed by Moldflow. In the original model of the correction tape box compounding cavity, the time unbalance rate of the melt in the mold cavity is about $28.6 \%$ and the pressure unbalance ratio is about $42.0 \%$. The original runner system is not balanced. The runner system is optimized through the filling analysis module and flow runner balance module of Moldflow. After optimization, the flow unbalance ratio changes from $28.6 \%$ to $0.7 \%$. The optimized runner system is balanced.

\section{The Geometric and Mesh Model of the Correction Tape Box}

The geometric model of the upper cover of the correction tape box is shown in Figure 1. The geometric model of the lower cover of the correction tape box is shown in Figure 2.

The models are then imported to Moldflow software by STL format. The meshed models of the upper and lower of the correction tape box are shown in Figure 3 and Figure 4. The plastic material used for the products is GE Plastics (Europe) ABS. The thicknesses of the upper and lower of the correction tape box are $1.5 \mathrm{~mm}$. The dimensions of the upper and lower covers of the correction tape box are all $125 \mathrm{~mm} \times 60 \mathrm{~mm} \times 1.5 \mathrm{~mm}$. There are three mesh types in Moldflow including Midplane, 3D and Fusion. Because of the thicknesses of the products, the Fusion mesh is adopted.

The mesh statistical chart of the upper and lower cover is shown in Table 1.

The mesh models of the upper and lower covers satisfy the demand of the runner balance analysis in Moldflow.

\section{Best Gate Location Analysis of the Correction Tape Box}

The shape of the upper and lower cover of the correction tape box is different, and the compounding cavity is asymmetric. Therefore, before designing the layout of the compounding cavity, it is necessary to use the best gate location analysis to find the best gate position of the upper and lower cover, and to

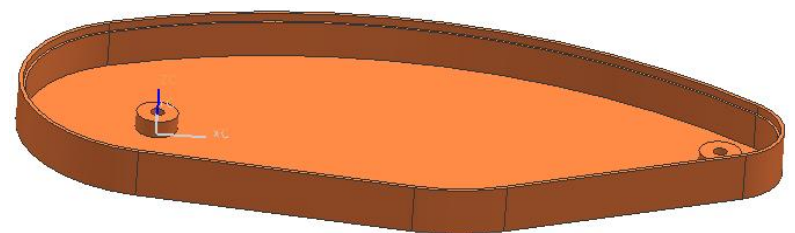

Figure 1. The geometrical model of the upper cover. 


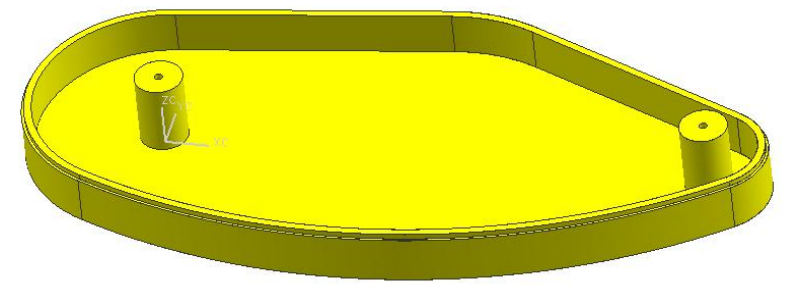

Figure 2. The geometrical model of the lower cover.

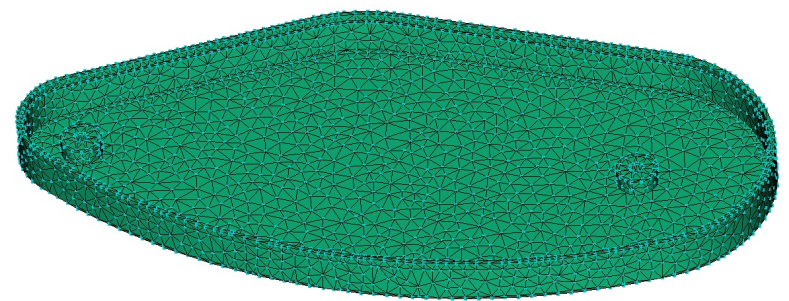

Figure 3. The mesh model of the upper cover.

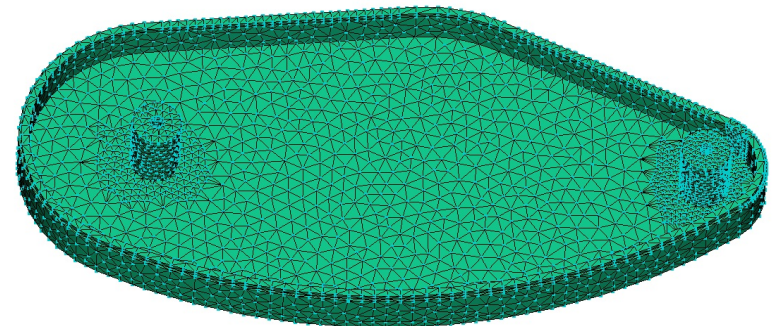

Figure 4. The mesh model of the lower cover.

Table 1. The mesh statistical chart of the upper and lower cover.

\begin{tabular}{ccc}
\hline & The upper cover & The lower cover \\
\hline Surface triangles & 8824 & 11,070 \\
Nodes & 4410 & 5537 \\
Connectivity regions & 1 & 1 \\
Surface area/cm ${ }^{2}$ & 121.023 & 126.319 \\
Aspect ratio & Maximum 6.0, Average 2.06, & Maximum 5.9, Average 1.91, \\
& Minimum 1.16 & Minimum 1.16 \\
Edge details & Free edges 0, Manifold edges & Free edges, Manifold edges \\
& 13236, Non-manifold 0 & 13236, Non-manifold 0 \\
Orientation details & Element not oriented 0 & Element not oriented 0 \\
Intersection details & Element intersections 0, & Element intersections 0, \\
Match ratio & Duplicate beam 0 & Duplicate beam 0 \\
92.2\% & $91.4 \%$
\end{tabular}

ensure the reasonable flow of melt in the single cavity, so as to provide a basis for further design of the compounding cavity. The best gate location of the upper cover is shown in Figure 5 and the best gate location of the lower cover is shown in Figure 6. 


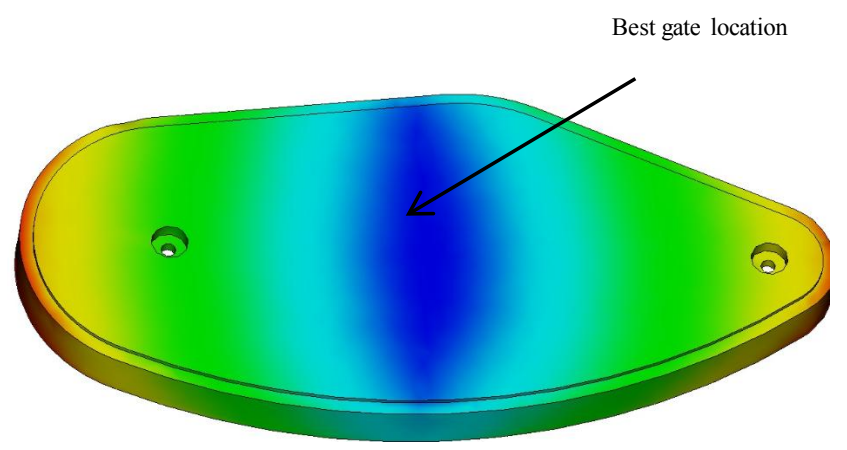

Figure 5. The best gate location of the upper cover.

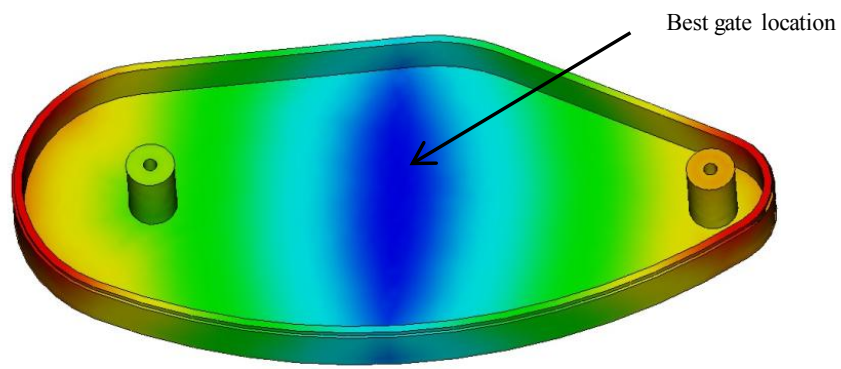

Figure 6. The best gate location of the lower cover.

\section{Initial Fill Analysis of the Compounding Cavity}

The runner balance analysis of the compounding cavity of the upper and lower cover needs to be based on the initial design scheme. Firstly, an original design scheme should be designed. Then the problems in the design scheme would be found out and adjusted. The needed analysis parameters and restrict conditions would be provided for the next runner balance analysis. The initial design scheme of the runner system for the compounding cavity of the correction tape box is shown in Figure 7. The initial dimensions of diversion channels leading to the upper and lower cover of the soap box are all $5 \mathrm{~mm}$. According to the flow situation of the single cavity provided by the best gate analysis, the gate is located in the middle of the upper and lower cover, and the side gate is adopted. The cross-sectional dimensions of the side circle gates are that start diameter $3.5 \mathrm{~mm}$ and end diameter $1.5 \mathrm{~mm}$.

The filling analysis is carried out to know the filling state of the flow in the compounding cavity, the pressure change state and the quality of the product after the filling process. The process parameters are set as follows: die surface temperature $60^{\circ} \mathrm{C}$, melt temperature $230^{\circ} \mathrm{C}$.

Through filling analysis, it can be found whether the melt flow in the compounding cavity is balanced or not, and the pressure changes during the flow process.

Filling time is shown in Figure 8 and we can find out the balance characteristic of the flow. As shown in Figure 8 the filling time of the upper cover is $1.067 \mathrm{~s}$ and the filling time of the lower cover is $0.781 \mathrm{~s}$. So the flow time unbalance ratio 


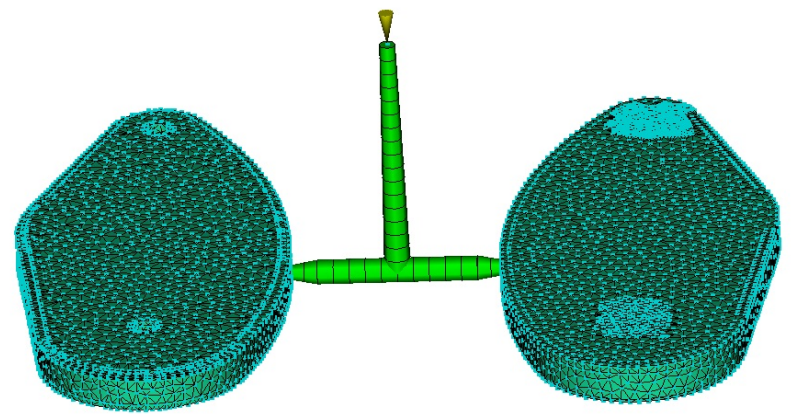

Figure 7. The initial runner system.

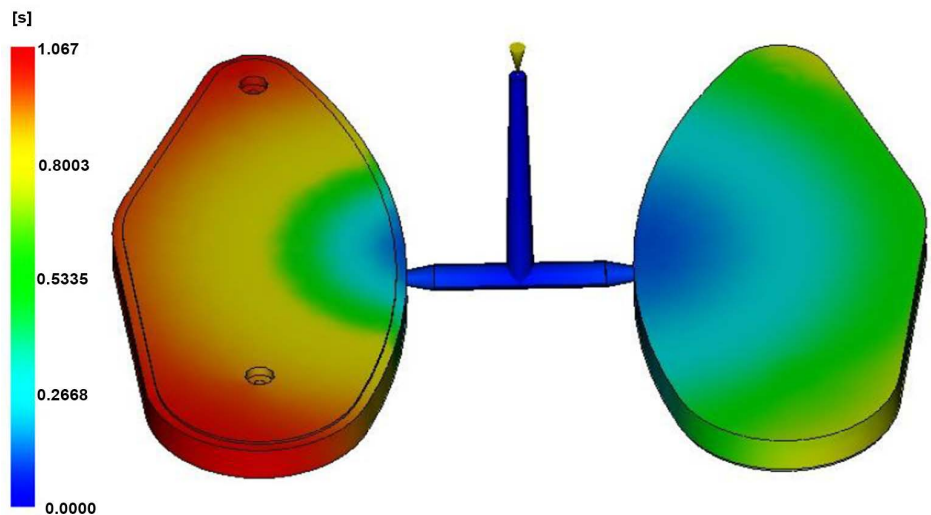

Figure 8. The injection time.

is about $14.1 \%$ and result in the pressure distribution unbalance in the two cavities. This would influence the quality of the product.

Pressure at V/P switchover is shown in Figure 9. The pressure at V/P switchover of the injection location is $73.17 \mathrm{MPa}$. From Figure 9 the lower is filled fully early and the pressure in the cavity is very high (the end pressure also attains $44.73 \mathrm{MPa}$ ). This condition results in over laden pack and influence the quality of the product.

Through the filling analysis of the initial design scheme of the compounding cavity of the upper and lower cover, it can be seen that the flow channel of the melt is unbalanced in the die cavity, and the time unbalance rate is $28.6 \%$.

Under the influence of the flow unbalance, the pressure difference between the upper and lower cover is quite large, and the pressure unbalance is $42.0 \%$. Next, the runner balance analysis of the compounding cavity is carried out to reduce the time and pressure unbalance rate.

\section{The Runner Balance Analysis of the Compounding Cavity}

By optimizing the compounding cavity, the time and pressure imbalance of melt flow in the cavity can be improved, the pressure difference between the upper and lower cavity can be reduced, and the product quality can be improved.

Iterative calculation parameters and convergence target settings are shown in Table 2. When setting iteration parameters, they can be adjusted by iterative calculation to obtain ideal parameters. 


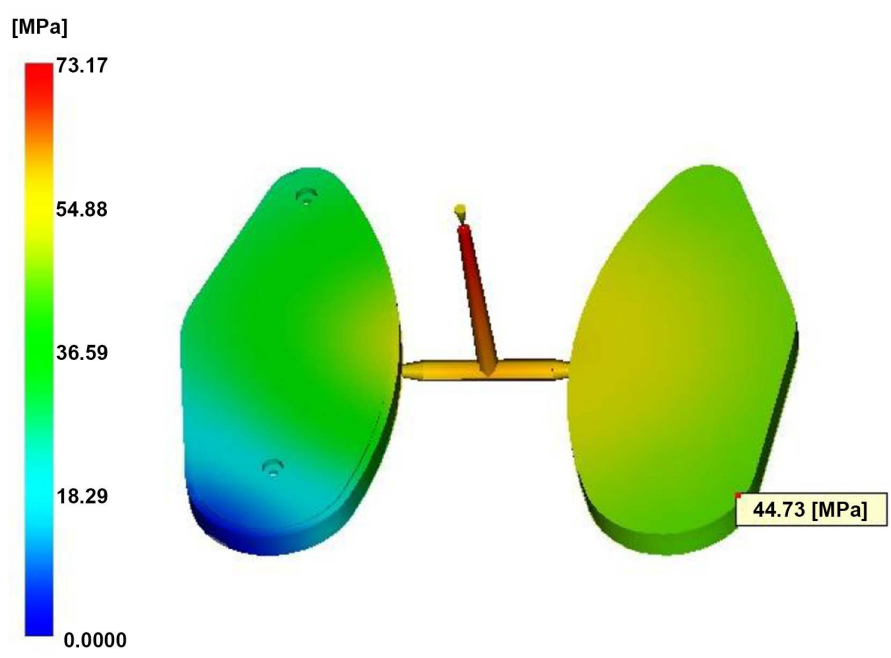

Figure 9. V/P change pressure.

Table 2. The iterative parameters setup.

\begin{tabular}{cc}
\hline Iterative parameters & Value \\
\hline Mill tolerance & 0.1 \\
Maximum iterations & 20 \\
Time convergence tolerance & 5 \\
Pressure convergence tolerance & 5 \\
\hline
\end{tabular}

After 13 iterations, the objective value converges, the convergence process of time imbalance rate is shown in Figure 10, and the pressure imbalance rate is shown in Figure 11. From Figure 10, it can be seen that the time and pressure imbalance rate have been significantly improved by optimizing the calculation. The time imbalance rate has been reduced from $28.6 \%$ to $0.7 \%$, and the pressure imbalance rate has been reduced from $42.0 \%$ to $4.2 \%$.

The size change of the optimized combined cavity runner is shown in Figure 12. As can be seen from Figure 12, the diameter of the diversion channel connecting the upper cover is almost unchanged, and the diameter of the diversion channel connecting the lower cover is reduced by $95.16 \%$. The diameter of the diversion channel connecting the lower cove is changed from $5 \mathrm{~mm}$ to $0.25 \mathrm{~mm}$.

From the runner balance analysis results, the runner balance optimization has achieved good results.

\section{Conclusions}

Aiming at the imbalance of flow in the compounding cavity of the upper and lower cover of correction tape box, Pro/e and Moldflow software were used to optimize the runner balance. Through optimization calculation, the time imbalance rate in the flow passage of the mould cavity decreased from $28.6 \%$ to $0.7 \%$, and the pressure imbalance rate decreased from $42.0 \%$ to $4.2 \%$. The pressure distribution in the cavity during the whole injection process was relatively uniform, and the optimization achieved very good results. 


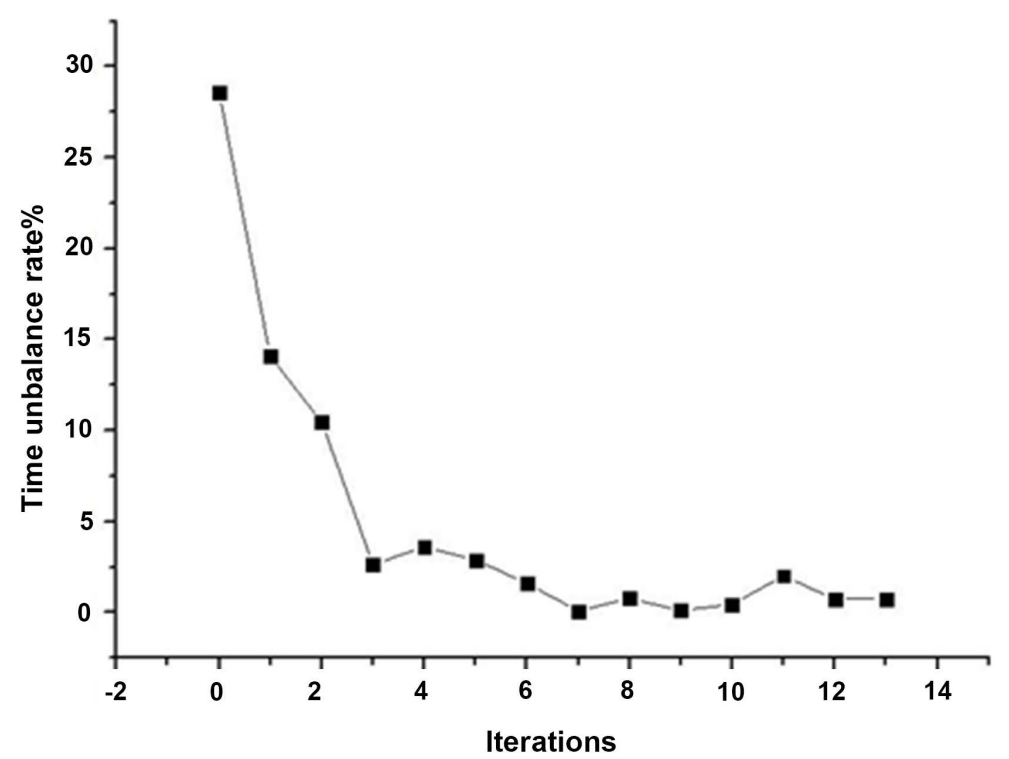

Figure 10. The time unbalance optimization process.

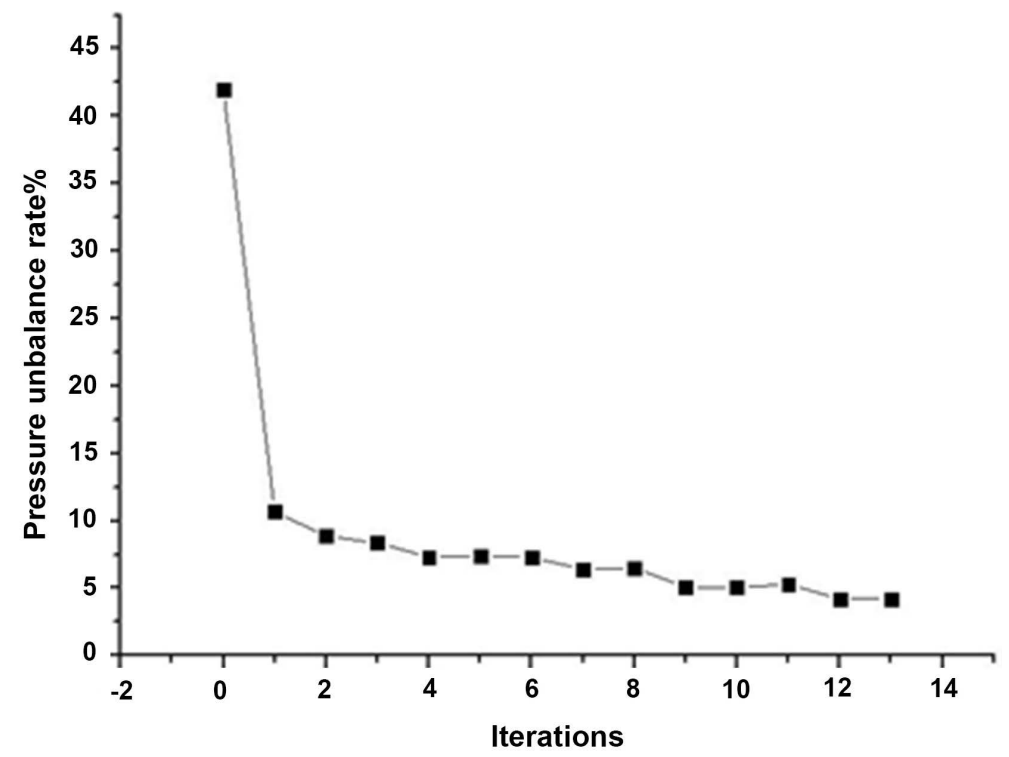

Figure 11. The pressure unbalance optimization process.

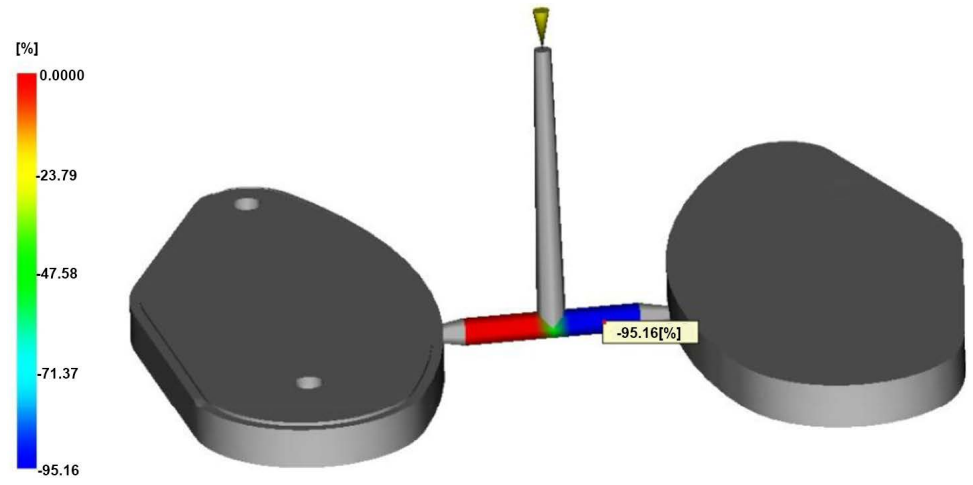

Figure 12. The size change of the optimized combined cavity runner. 
The research shows that the injection mold with compounding cavity can realize the melt flow balance by runner balance optimization, which can effectively improve the molding quality of the products and improve the production efficiency. The results provide a useful reference for researchers engaged in die design.

\section{Acknowledgements}

This work supported by Suzhou science and technology planning project (SYG201823); Suzhou vocational university research project (SVU2018YY07); sponsored by Qing Lan Project.

\section{Conflicts of Interest}

The authors declare no conflicts of interest regarding the publication of this paper.

\section{References}

[1] Hong, J.C. (2010) Flow Balance Optimization of Different Cavities of Mold Based on Moldflow Software. Engineering Plastics Application, 38, 35-38.

[2] Du, J. (2018) Optimization Analysis of a Soap Box Combined Cavity Flow Balance Based on Moldflow. Journal of Electroceramics, 46, 72-76.

[3] Park, H.-S. and Dang, X.-P. (2010) Optimization of Conformal Cooling Channels with Array of Baffles for Plastic Injection Mold. International Journal of Precision Engineering and Manufacturing, 117, 879-890. https://doi.org/10.1007/s12541-010-0107-Z

[4] Vishnuvarthanan, M., Panda, R. and Ilangovan, S. (2013) Optimization of Injection Molding Cycle Time Using Moldflow Analysis. Middle-East Journal of Scientific Research, 13, 944-946. 